\title{
Measurement Invariance Assessment with Bayesian Hierarchical Inclusion Modeling
}

\author{
Stephen R. Martin, Donald R. Williams, Philippe Rast \\ University of California, Davis
}

\begin{abstract}
Measurement non-invariance is a common concern among Psychologists and other researchers who measure unobservable constructs. Failing to assess or adjust for the presence of non-MI can produce spurious differences and relationships, and yield inaccurate predictions. Random effects (RE) models have successfully been used to model non-invariance, but the ability to accept invariance can be improved. This paper extends the RE model with a hierarchical structure on the RE variances. By doing so, $\mathrm{RE}$ variances can be effectively set to zero, and invariance information is shared across dependent parameters. A simulation confirms that the extended model (HM-RE) increases the ability to make decisions about invariance and non-invariance, while maintaining estimation accuracy. An example using real data is provided.
\end{abstract}

Keywords: Measurement invariance, Bayesian, Random effects, Bayes factor

Most psychological research questions operate under the premise that we can measure certain latent constructs and that we can meaningfully compare and interpret differences in these latent constructs across groups, time, or conditions. Before engaging in meaningful comparisons across groups, we need to test the implicit assumption that the same construct is being measured; that is, we need to ensure that we obtain measurement invariance (MI) or equivalence. From a conceptual point of view, MI exists if individuals with equal latent scores have the same probability of responses, regardless of group membership (Meredith, 1993). If MI does not hold, differences in latent factors can not be attributed unequivocally to group differences in the underlying construct, as they may arise due to differences in the way the measurements themselves operate within groups. Conversely, when MI holds, latent comparisons are valid across groups.

Currently, the most common approach to investigating

Corresponding author:

Stephen R. Martin

Department of Psychology

University of California Davis

srmart@ucdavis.edu

Research reported in this publication was supported by the $\mathrm{Na}-$ tional Institute On Aging of the National Institutes of Health under Award Number R01AG050720 to PR. The content is solely the responsibility of the authors and does not necessarily represent the official views of the National Institutes of Health.
MI is in the context of CFA or IRT models that derive latent factors from multiple underlying items. To date, the standard approach to establishing MI consists of a series of tests that sequentially adds or removes constraints to a multigroup factor analytic measurement model. For example, model comparison starts at the configural level, in which a basic factor structure, defined by the the same set of items and latent factors, is imposed across different groups. Once configural invariance holds, additional constraints are added consecutively to equate loadings (weak invariance), intercepts (strong invariance), and residual variances (strict invariance) across groups (cf. Cheung \& Rensvold, 1999). After each step, the more constrained model is compared to the previous one via a $\chi^{2}$-difference test or critical differences in model fit indices, such as in AIC and RMSEA (Cheung \& Rensvold, 2002). If the more constrained model does not fit the data significantly worse than the less constrained, MI at that constrained level is said to be achieved. There is general agreement that at least strong invariance needs to be established in order to make meaningful comparisons among factor scores (Meredith \& Teresi, 2006).

\section{Random Effects Approach}

While MI has mostly been addressed by performing a sequence of significance tests, an alternative approach that is gaining popularity addresses MI by utilizing a random effects framework. As the name suggests, measurement invariance exists if the variance in measurement parameters is null or negligible. Hence, a direct approach to assessing invariance is to model this variance. Random effect (RE) models are well-suited for this task, and have been success- 
fully used to assess differential item functioning in item response theory frameworks (de Jong \& Steenkamp, 2010; de Jong, Steenkamp, \& Fox, 2007). Whether weak, strong, or strict invariance exists can be directly determined from the variance estimates of the respective parameters. The aim of this paper is to extend the RE approach so that invariance is easier to detect, and invariance information is shared across similar parameters.

The RE approach has gained traction in recent years, as it offers several advantages over the classical approach. For one, it scales well with the number of groups over which invariance is tested and it can accommodate small group sample sizes due to partial pooling. Also, no invariant referent items are required for identification, which can have a substantial impact on the ability to assess invariance (Meade \& Wright, 2012; Meredith \& Teresi, 2006; Steenkamp \& Baumgartner, 1998; de Jong et al., 2007). Moreover, the $\mathrm{RE}$ approach jointly models the variance of all parameters, which reduces the analytic flexibility particularly when assessing partial invariance (Gelman \& Loken, 2013). Pairwise comparisons can be computed from the random effect estimates of each group. Despite a large number of possible comparisons, the hierarchical shrinkage of the estimates reduces the probability of drawing erroneous conclusions (Gelman, Hill, \& Yajima, 2012). Further, due to the joint estimation of factor scores and measurement variance, latent inferences are adjusted for the presence of measurement non-variance. This important feature permits researchers to make meaningful latent inferences despite the presence of non-invariance.

Although the RE model has these desirable features, there is still room for improvement. In particular, the RE model makes two assumptions that complicate invariance assessment. First, it assumes that at least a small amount of variance exists in every parameter. This assumption directly conflicts with the goal of accruing evidence in favor of invariance. Ideally, the RE model should allow strong evidence in favor of zero variance, and collapse to a fixed effect model when full invariance is plausible. Second, it assumes that parameter invariance is independent of all other parameters. However, parameters share characteristics that can further inform whether invariance exists. For example, if an item is interpreted differently among groups, then all related parameters for that item may be non-invariant. More specifically, if a factor loading for an item exhibits variability across groups, then its corresponding intercept and residual may be more likely to exhibit non-invariance. Consequently, non-invariance in the item's loading should inform whether the item's intercept and residual variance are also non-invariant.

In the present work we present a model that overcomes the limitations in the classic RE model by adding hierarchical structure to the variance parameters of interest. The goal is to define a RE model in which the variance among groups can be effectively zero (invariant), or nonzero and permitted to manifest accordingly (non-invariant). Whether a parameter is invariant should be informed by the invariance of parameters with shared characteristics (e.g., parameters that operate on the same item, parameters of the same class). To implement these improvements, the proposed model imposes an additional hierarchical structure that permits variance to be effectively zero or non-zero, and is therefore termed a hierarchical inclusion model (HM-RE) on the random effect variances. By adding parameter characteristics to the hierarchical inclusion model, invariance information is pooled across parameters, and the ability to discriminate non-invariance from invariance among groups can be improved. While maintaining all of the benefits of an RE model, the HM-RE extension should permit greater information to be extracted from the data resulting in a higher number of accurate decisions regarding measurement invariance.

For the purpose of this paper, we focus on a unidimensional, normal-assumptive model without residual covariances. The proposed model is based on the random effect approach, described in detail first. Due to the extended hierarchical model on parameter priors, a fully Bayesian approach is required. The priors and identification constraints are detailed, followed by the proposed hierarchical extension. An approach for testing MI is described, and a simulation is performed to evaluate the extended model. Finally, we use the model to assess MI in an open personality dataset.

\section{Model Description}

The proposed method extends the random effects approach with a hierarchical inclusion model on the random effect variances. We first describe the random effects model here.

\section{Random Effect Model}

Let $y_{i j}^{k}$ be the observation of individual $i \in 1 \ldots N$ of group $k \in 1 \ldots K$ on item $j \in 1 \ldots J$, such that

$$
y_{i j}^{k} \sim \mathcal{N}\left(v_{j}^{k_{i}}+\lambda_{j}^{k_{i}} \eta_{i}, \sigma_{j}^{k_{i}}\right) .
$$

Hence, each observation $y_{i j}^{k}$ is assumed to be from a normal distribution with the mean defined as a linear combination of the intercept, $v$, the factor score, $\eta$, its corresponding loading $\lambda$, and residual standard deviation given by $\sigma$ for each group $k_{i}$. For the sake of simplicity, assume a unidimensional factor model, with latent factor scores $\boldsymbol{\eta} \sim \mathcal{N}\left(\alpha^{k}, \sigma_{\eta}^{k}\right)$ where $\alpha^{k}$ is the factor mean for each group and $\sigma_{\eta}^{k}$ defines the factor variance for each group.

Note that the group-specific parameters $\left(\lambda^{k}, \sigma^{k}\right.$, and $\left.v^{k}\right)$ 
are defined as the sum of a fixed and a random effect - I.e.,

$$
\begin{aligned}
\lambda^{k} & =\lambda+\boldsymbol{u}_{\lambda}^{k} \\
\log \left(\boldsymbol{\sigma}^{k}\right) & =\boldsymbol{\sigma}^{*}+\boldsymbol{u}_{\sigma}^{k} \\
\boldsymbol{v}^{k} & =\boldsymbol{v}+\boldsymbol{u}_{v}^{k} .
\end{aligned}
$$

Greek symbols without superscripts denote the fixed effects and the $\boldsymbol{u}^{k}$ 's are the random effects. $\boldsymbol{\sigma}^{*}$ is the fixed effect of the residual standard deviation with its corresponding random effect $\boldsymbol{u}_{\sigma}^{k}$ on the log scale. We then assume that the random effects, for the loadings, intercepts, and residual standard deviations, are drawn from a common distribution. In other words, the three random effect vectors of length $J$ for each group are then stacked into a vector of length $3 J$ and distributed multivariate normally such that

$$
\left[\boldsymbol{u}_{\lambda}^{k}, \boldsymbol{u}_{\sigma}^{k}, \boldsymbol{u}_{v}^{k}\right]^{\prime} \sim \mathcal{N}(\mathbf{0}, \Sigma)
$$

This implements a basic hierarchical model in which groupspecific parameters are partially pooled and allowed to correlate according to the $3 J \times 3 J$ covariance matrix $\boldsymbol{\Sigma}$ for the three sets of parameters. It is important to note that the random effects for both the mean structure and the scale are coming from a common multivariate distribution. Hence, random effects for the residual variance are allowed to correlate with random effects for the mean structure, or the location. This approach has gained popularity in recent years and is known as the mixed effects location scale model (Hedeker, Mermelstein, \& Demirtas, 2008; Rast, Hofer, \& Sparks, 2012; Rast \& Ferrer, 2018; Williams, Liu, Martin, \& Rast, 2019).

Following the recommendation from Barnard, McCulloch, and Meng (2000), we employ a separation strategy for estimating the covariance matrix - i.e.,

$$
\Sigma=D L L^{\prime} D
$$

Here $\boldsymbol{D}$ is a $3 J \times 3 J$ diagonal matrix that contains the random effects standard deviations $\left(\sigma_{\lambda}, \sigma_{\sigma}, \sigma_{v}\right)$ and a Choleskydecomposed correlation matrix $(\boldsymbol{L})$ of the same dimension. This formulation has several advantages. Namely, it facilitates computation and allows one to define separate priors for the standard deviations and correlations. This permits additional structure to the random effect standard deviations, the parameters of primary interest in MI assessment.

Priors and Identification. So far, we have introduced the standard random effects model. Next, we will expand that model with specific prior distributions that are both reasonable and identify the model. The priors on the residual SD's and intercepts are weakly informative (for approximately standardized data) and defined as follows:

$$
\begin{aligned}
\sigma^{*} & \sim \mathcal{N}(0,1) \\
\boldsymbol{v} & \sim \mathcal{N}(0,1) .
\end{aligned}
$$

The sign of the loadings and latent variables are unidentifiable without further constraints. In order to identify the direction of a standardized latent variable, a positivity constraint is typically imposed on the loadings. However, the multilevel framework complicates such a constraint. In particular, constraining fixed loadings (and not the groupspecific loadings) to be positive is insufficient, and can result in a multimodal posterior with two solutions. One solution will manifest as fixed loadings being near zero (i.e., as close to negative as possible), with high random effect variance that permits group-specific loadings to be negative, and the latent scores to be reversed. The second solution, which is the target solution, will manifest as fixed loadings being notably positive, such that group-specific loadings will largely be positive, and the latent scores in the intended direction. This complicates estimation, especially with MCMC, because different chains may either gravitate toward one of the two different solutions, or each chain will oscillate between the two solutions. In either case, the resulting posterior estimates are misleading.

In order to ensure a reasonable, unimodal solution, we impose a constraint to ensure that all group-specific loadings are at least zero. That is, we impose a constraint to ensure that $\lambda_{j}+u_{\lambda j}^{k} \geq 0$, for all $u_{\lambda j}^{k}$. This condition is sufficiently met when $\lambda_{j}+\min _{k} u_{\lambda j}^{k} \geq 0$. Solving for this, the lower bound for the fixed loading for item $j$ is therefore $\mathrm{LB}_{j}=-\min _{k} u_{\lambda j}^{k}$. The fixed loading is assigned a standard half-normal prior, further truncated at this lower bound $\mathrm{LB}_{j}$. The prior for the fixed effect is therefore defined as:

$$
\begin{aligned}
p\left(\lambda_{j}\right) & =\frac{\mathcal{N}^{+}\left(\lambda_{j} \mid 0,1\right)}{1-\Phi^{+}\left(\mathrm{LB}_{j}\right)}, \lambda_{j}+u_{\lambda j}^{k} \geq 0 \\
\mathrm{LB}_{j} & =-\min _{k} u_{\lambda, j}^{k},
\end{aligned}
$$

where $\Phi^{+}$is the CDF for the half-normal.

Latent scores are normally distributed, with groupspecific means and variances. In order for them to be identifiable, constraints are required. Here, we constrain the means and log SD's to sum to zero. This preserves the relative differences between groups, and keeps latent variables on a meaningful scale. Formally,

$$
\begin{aligned}
\boldsymbol{\eta} & \sim \mathcal{N}\left(\alpha^{k_{i}}, \sigma_{\eta}^{k_{i}}\right) \\
\sum_{k} \alpha^{k} & =0 \\
\sum_{k} \log \sigma_{\eta}^{k} & =0 .
\end{aligned}
$$

As described in Equation 1, the covariance matrix $\boldsymbol{\Sigma}$ of the random effects was defined by the Cholesky-decomposed correlation matrix $\boldsymbol{L}$ and the diagonal matrix $\boldsymbol{D}$ containing the random effects SD's. The correlation matrix is composed 


$$
\log \theta_{p}=\tau+\text { Item }_{j_{p}}+\text { Parameter }_{P_{p}}+\delta_{p}
$$

$$
\sigma_{p} \sim \mathcal{N}^{+}\left(0, \theta_{p}\right)
$$

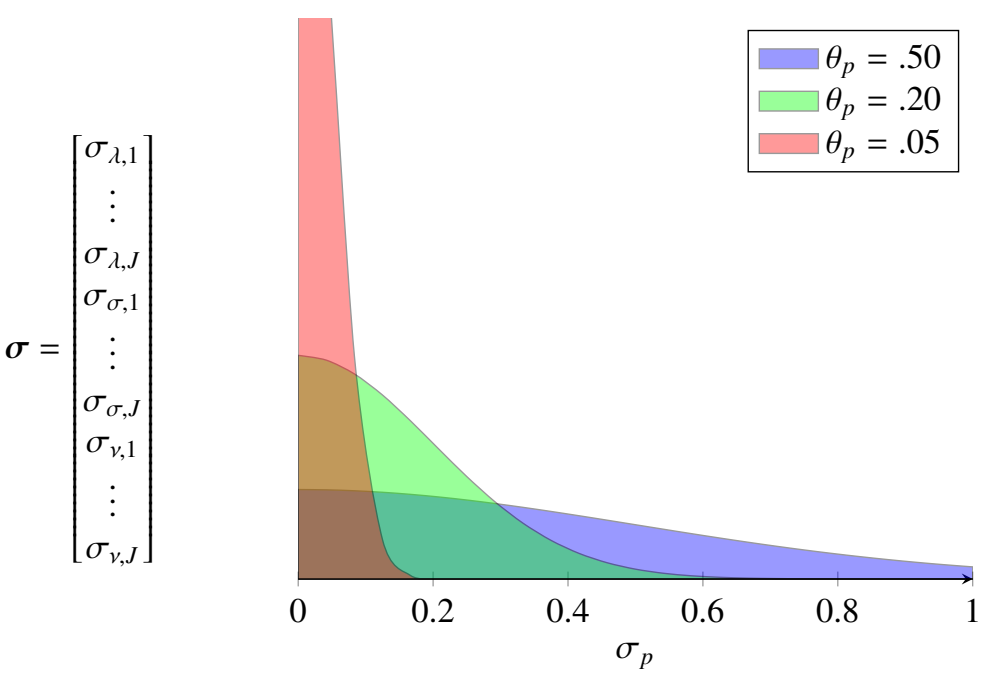

Figure 1. The hierarchical parameter inclusion model for the group variances may manifest as depicted. If a particular item or parameter class exhibits variability, the probability of variability increases for other parameters from that item or other parameters within the same class. That is, variance in an item's parameter $\left(\sigma_{P, j}\right)$ affects the corresponding Item $j$ and Parameter $_{P}$ values, which in turn affects $\theta_{p}$ for other parameters, and therefore the probability of variance elsewhere.

and assigned the LKJ prior - I.e.,

$$
\boldsymbol{L} \boldsymbol{L}^{\prime} \sim \operatorname{LKJ}(\eta=3)
$$

The LKJ prior (Lewandowski, Kurowicka, \& Joe, 2009) is a spherical prior on correlation matrices. More mass is placed on the identity matrix as $\eta$ increases, with $\eta=1$ defining a uniform joint distribution.

The random effect standard deviations $(\sigma)$, of primary interest in MI assessment, are contained within the diagonal of $\boldsymbol{D}\left(\sigma_{p}=\boldsymbol{D}_{p p}\right)$. Each standard deviation, $\sigma_{p} \in \boldsymbol{\sigma}$, is assigned a positive half-normal prior with a corresponding scale, $\theta_{p} \in \boldsymbol{\theta}$ :

$$
\sigma_{p} \sim \mathcal{N}^{+}\left(0, \theta_{p}\right)
$$

Each $\theta_{p}$ in the 3J-length vector $\boldsymbol{\theta}$ is defined by the hierarchical inclusion model, described in the next section.

\section{Hierarchical Inclusion Model}

The first goal of the hierarchical inclusion model is to permit random effect variances to be effectively zero, and removed from the model. To accomplish this goal, the hierarchical inclusion model modifies the prior width, $\theta_{p}$ in $\boldsymbol{\theta}$, for each random effect standard deviation, $\sigma_{p}$ in $\sigma$.
Let $p \in 1 \ldots 3 J$ be the index of an element in $\sigma$ or $\boldsymbol{\theta}$. We organize both $\boldsymbol{\theta}$ and $\boldsymbol{\sigma}$ such that the first $J$ entries correspond to the items' loadings, the second $J$ to residual variances, and the third $J$ to intercepts. For example, $\theta_{1}$ is the prior width for the random effect standard deviation $\sigma_{1}$, which corresponds to $\sigma_{\lambda, 1}$. Hence, if a given $\theta_{p}$ is small, then the random effect standard deviation of the corresponding parameter, $\sigma_{p}$, is given a prior distribution with most of its mass concentrated at or near zero. The standard deviation is therefore given a high probability of being effectively zero, and the parameter is rendered effectively invariant. Conversely, if a given $\theta_{p}$ is large, then $\sigma_{p}$ is given a wide prior distribution, and the standard deviation has a high probability of being non-zero, or being non-invariant. In essence, by allowing $\theta_{p}$ to vary, the prior probability of invariance can be altered (See Figure 1).

The second goal is to permit invariance of one parameter to alter the prior probability of invariance in other parameters with shared characteristics. $\boldsymbol{\theta}$ contains elements that do not need to be considered independent. For example, if one item has variable intercepts, then the prior probability of variance in the other two parameters (i.e., the item's loading and residual variance), should be increased as well. These 
parameters model a common item, and if an item operates differently across groups, then its corresponding parameters should all have a jointly higher probability of variance. Similarly, if one item has a variable loading, then the probability of non-invariance in other items' loadings should increase. Other shared item characteristics can be included (e.g., if a subset of items use particular wordings, that may act as a predictor of variance inclusion).

In order to encode these goals, each $\log \left(\theta_{p}\right)$ is hierarchically modeled as the sum of four parameters:

$$
\log \left(\theta_{p}\right)=\tau+\text { Item }_{j_{p}}+\text { Parameter }_{P_{p}}+\delta_{p}
$$

where $\tau$ is a fixed effect parameter for the width of the priors on the log scale. Item-wide parameters and class-wide parameters inform one another through the corresponding Jlength Item and 3-length Parameter vectors, respectively. Item $_{j_{p}}$ is the effect of the item corresponding to $\theta_{p}$, and Parameter $_{P_{p}}$ is the effect of the parameter type corresponding to $\theta_{p}$.

More explicitly, Equation (2) can be expanded to

$$
\left[\begin{array}{c}
\log \theta_{1} \\
\log \theta_{2} \\
\vdots \\
\log \theta_{J+1} \\
\log \theta_{J+2} \\
\vdots \\
\log \theta_{3 J}
\end{array}\right]=\left[\begin{array}{c}
\tau \\
\tau \\
\vdots \\
\tau \\
\tau \\
\vdots \\
\tau
\end{array}\right]+\left[\begin{array}{c}
\text { Item }_{1} \\
\text { Item }_{2} \\
\vdots \\
\text { Item }_{1} \\
\text { Item }_{2} \\
\vdots \\
\text { Item }_{J}
\end{array}\right]+\left[\begin{array}{c}
\text { Parameter }_{\lambda} \\
\text { Parameter }_{\lambda} \\
\vdots \\
\text { Parameter }_{\sigma} \\
\text { Parameter }_{\sigma} \\
\vdots \\
\text { Parameter }_{v}
\end{array}\right]+\left[\begin{array}{c}
\delta_{1} \\
\delta_{2} \\
\vdots \\
\delta_{J+1} \\
\delta_{J+2} \\
\vdots \\
\delta_{3 J}
\end{array}\right]
$$

For example, if item $j$ has variance in the intercept and loading, then Item $_{j}$ can increase, and the prior probability of variance for the remaining parameter, the residual variance, increases. Similarly, if several items have invariant intercepts, then Parameter $_{v}$ can decrease, and the prior probability of invariance in other intercepts will increase. If all items and parameters are invariant, then $\tau$ alone can decrease, and the prior probability of invariance in all parameters will increase. The structure therefore pools information about the presence or absence of invariance across items and parameters. Instead of requiring that $3 \mathrm{~J}$ variances overcome their prior to support invariance, merely one, three, or J parameters need to change for invariance to effectively manifest. Finally, the 3J-length vector, $\boldsymbol{\delta}$, enables specific $\sigma_{p}$ 's to manifest and escape otherwise high prior mass at zero when the data favor doing so. For example, Parameter $\lambda$ and Item $_{1}$ may both be low because loadings are generally invariant, and the intercept and residual variance are invariant for item 1. Combined, this would put substantial mass over zero for $\sigma_{\lambda, 1}$, making it difficult to detect non-invariance. Therefore, if the data necessitate non-invariance in $\lambda_{1}$, then $\delta_{1}$ can increase and permit a wider prior for that specific random effect variance.
The priors for the continuous hierarchical parameter inclusion model for the parameter variances are defined as

$$
\tau \text {, Item, Parameter, and } \delta \sim \mathcal{N}\left(0, \sigma_{\tau}\right) \text {, }
$$

where the hyperparameter, $\sigma_{\tau}$, is assumed to be 1 throughout this paper (i.e., a standard normal prior). ${ }^{1}$ Note that the hierarchical parameter inclusion model does not predict the RE standard deviations directly, but rather the scale of the prior for them (See Figure 1).

\section{Decision Rules}

The HM-RE enhances discriminability between the presence or absence of invariance by permitting posterior mass to concentrate at zero or outside of zero. However, a formal decision rule is still desired when the goal is to determine which items and parameters are invariant. Because the question of invariance assessment is whether measurement parameters vary across groups, a decision can be made on whether the standard deviation of the parameter, $\sigma_{p}$, is zero. Given that our model is specified in a Bayesian framework, Bayes factors $(\mathrm{BF})$ can be obtained to quantify evidence in favor of invariance or non-invariance (A. J. Verhagen \& Fox, 2012; J. Verhagen, Levy, Millsap, \& Fox, 2016).

The Bayes factor is defined as the ratio of marginal likelihoods of two hypotheses, $H_{0}$ and $H_{1}$. Let $H_{0}$ be the hypothesis that $\sigma_{p}=0$, and $H_{1}$ that $\sigma_{p}>0$, respectively encoding invariance and non-invariance. If $\mathrm{BF}_{01}$ exceeds an evidentiary threshold, the data favor $H_{0}$, or invariance. We set the threshold to 6 in this paper. Conversely, if $\mathrm{BF}_{01}$ is below the reciprocal, $\frac{1}{6}$, then non-invariance is supported. No decision is made when the BF favors neither invariance nor non-invariance.

The BF can be computed on each parameter standard deviation. However, marginal likelihoods are notoriously difficult to compute, especially when several models are considered. One simple method, appropriate for nested models, is the Savage-Dickey density ratio (Dickey \& Lientz, 1970; Wagenmakers, Lodewyckx, Kuriyal, \& Grasman, 2010)

$$
\begin{aligned}
B F_{01} & =\frac{p\left(\boldsymbol{Y} \mid H_{0}\right)}{p\left(\boldsymbol{Y} \mid H_{1}\right)} \\
& =\frac{p\left(\sigma_{p}=0 \mid \boldsymbol{Y}, H_{1}\right)}{p\left(\sigma_{p}=0 \mid H_{1}\right)} .
\end{aligned}
$$

The ratio of posterior and prior density at $\sigma_{p}=0$ provides the Bayes factor in favor of invariance.

The prior for each $\sigma_{p}$ is amenable to this approach, but must be marginalized over the hierarchical inclusion model.

${ }^{1}$ The priors may be freely specified for each component, but identical hyperparameters simplify the marginal prior expression. 
The prior for $\sigma_{p}$ using the above prior (Equation 4) then is:

$$
\begin{aligned}
p\left(\sigma_{p}\right) & =\int \mathcal{N}^{+}\left(\sigma_{p} \mid 0, \theta_{p}\right) \log \mathcal{N}\left(\theta_{p} \mid 0, \sqrt{4 \sigma_{\tau}^{2}}\right) d \theta_{p} \\
& =\int \frac{1}{2 \theta_{p}^{2} \sigma_{p} \pi} \exp \frac{-4\left(\sigma_{\tau} \sigma_{p}\right)^{2}-\left(\theta_{p} \log \theta_{p}\right)^{2}}{8 \theta_{p}^{2} \sigma_{\tau}^{2}} d \theta_{p}
\end{aligned}
$$

Approximating Equation (5) and the posterior density requires simulation or numerical approximations, detailed in the Estimation section.

Furthermore, whether non-invariance is present for a parameter may not be the only question of interest. The approach presented here allows one to examine pairwise differences between all groups for a parameter, and determine which differences are non-zero. To do so, a BF can be computed on any group difference in parameters, $u_{p}^{k}-u_{p}^{\urcorner k}$. Let $H_{0}: u_{p}^{k}-u_{p}^{\urcorner k}=0$ and $H_{1}: u_{p}^{k}-u_{p}^{\urcorner k} \neq 0$. Again, while the Savage-Dickey method can be used, the implied prior on the difference must be derived. Assuming that the random effects have the same normal prior with unknown variance, then the implied prior on the difference has twice the variance. Marginalizing over the unknown variance, the prior on pairwise differences is therefore defined as:

$$
\begin{aligned}
p\left(u_{p}^{k}-u_{p}^{\urcorner k}\right) & =\int \mathcal{N}\left(u_{p}^{k}-u_{p}^{\urcorner k} \mid 0, \sigma_{p} \sqrt{2}\right) p\left(\sigma_{p}\right) d \sigma_{p} \\
& =\int \frac{1}{2 \sigma_{p} \sqrt{\pi}} \exp \frac{-\left(u_{p}^{k}-u_{p}^{\urcorner}\right)^{2}}{4 \sigma_{p}^{2}} p\left(\sigma_{p}\right) d \sigma_{p} .
\end{aligned}
$$

As before, the present paper approximates this density as detailed in the next section.

\section{Estimation}

The HM-RE necessitates a Bayesian framework because the hierarchical inclusion model modifies prior probabilities. Because an analytic solution to the HM-RE is untenable, a markov chain monte carlo (MCMC) approximation is needed. The MCMC algorithm must be capable of efficiently sampling and navigating the high dimensional space and mixed effects models have proven to be challenging for classic methods such as Gibbs sampling, due to correlated random effects. In recent years, the No-U-TurnSampler (NUTS), an efficient variant of hamiltonian monte carlo (HMC), has gained popularity as it has proven particularly useful for sampling from high dimensional distributions and hierarchical models (Hoffman \& Gelman, 2011). The hierarchical inclusion model proposed here is continuous and therefore tractable with HMC, NUTS, and other gradient-based algorithms. Stan, a general purpose probabilistic programming package (Carpenter et al., 2017), implements NUTS and was used to estimate the HM-RE.
The $\mathrm{BF}$ for invariance is computed using the ratio of posterior and prior densities at $\sigma_{p}=0$. The prior density (Equation 5) at zero can be numerically approximated under a given $\sigma_{\tau}$; in this paper, $\sigma_{\tau}=1$, and $p\left(\sigma_{p}=0 \mid \sigma_{\tau}=1\right) \approx$ 5.8956. The posterior density can be approximated using density estimation on posterior samples. Because the posterior can have substantial mass at the lower boundary of zero, a lower-bounded logspline density estimator was chosen (Deng \& Wickham, 2011).

The BF for pairwise comparisons is likewise computed using the ratio of posterior and prior densities at $u_{p}^{k}-u_{p}^{\urcorner k}=$ 0 . Because the required integration is untenable, both the prior and posterior densities are approximated using a logspline density estimator on samples from each.

\section{Simulation}

Model Specification. The simulation compares the hierarchical inclusion (HM-RE) model with the random effects (RE) model. In order for the two to be maximally comparable, the RE model employs the implied marginal prior of the HM-RE model (Equation 5). This ensures that the only difference between the two model specifications is the presence or absence of the hierarchical inclusion structure.

With this prior specification, the BFs are directly comparable, and any difference between the models' performances is due to hierarchical inclusion structure on the prior, rather than differences in the prior, per se.

Data Generation. K groups are created with $n$ individuals in each. Latent scores are generated from a standard normal distribution. The number of items, J, was fixed to 10. Loadings are uniformly generated from .4 to .98. Intercepts are normally generated with mean zero and standard deviation of .2. Log Residual SDs are computed from $\log \sqrt{1-\lambda_{j}^{2}}$. Random effects for non-invariant parameters are normally distributed with mean zero and a standard deviation of $.4\left(\sigma_{p}^{2}=.16\right)$. The group-specific measurement model is constructed from the fixed and random effects, and data are generated using $y_{i j}^{k} \sim N\left(v_{j}^{k}+\eta_{i} \lambda_{j}^{k}, \sigma_{k}^{k}\right)$.

Simulation Conditions. Several conditions were created for simulation to cover a wide but realistic array of different scenarios. Three group sizes $(K \in 3,5,10)$, three within group sample sizes $(n \in 50,100,300)$, and six invariance conditions were combined to produce 54 possible conditions. The six invariance conditions are:

- Full invariance

- Non-invariance in five items

- Non-invariance in all parameters

- Non-invariance in loadings

- Non-invariance in (log) residual SDs 
- Non-invariance in intercepts

Each condition was replicated ten times. In each of 540 iterations, data were generated according to the condition, and the RE and HM-RE models were each fit to the generated data.

Simulation Statistics. The MSE, bias, and 95\% interval coverage rates were computed for the fixed parameters, random parameters, random effect standard deviations, and latent scores. The correlation of the latent scores with the true scores was additionally computed.

Finally, decisions about the presence or absence of invariance was computed using a Bayes factor on the parameter standard deviations for each parameter. The threshold was set to 6 or $1 / 6$ for sufficient evidence in favor of invariance and non-invariance, respectively. All BFs within that range were classified as "undecided". The true positive and negative rates, positive and negative predictive values, accuracies, and conditional accuracies were computed. The true positive rate (TP) is the proportion of all non-invariant parameters detected as non-invariant, and true negative rate (TN) is the proportion of invariant parameters detected as invariant. The positive predictive value is the proportion of parameters detected as non-invariant that are truly noninvariant, and negative predictive value is the proportion of detected invariant parameters that are truly invariant. Conditional accuracy refers to the accuracy of decisions given a decision was made, whereas accuracy refers to the proportion of correct decisions over all possible decisions.

\section{Results}

Two of the 540 iterations exhibited errors in the logspline estimator, and were reestimated with new data. Only one iteration demonstrated poor convergence $(\hat{R}>>1)$, and was reestimated with new data. All other iterations were successful.

The primary goal of the HM-RE model is to extend the RE model such that the random effect variances can essentially manifest, or not, using shared information from other items and parameters. Consequently, decision rates were expected to differ between the approaches. Conversely, the parameter estimates were expected to be similar between the approaches, given that the only difference between the models is the hierarchical inclusion model on the parameter variances.

Indeed, HM-RE and RE performed similarly in MSE and bias, with the exception of the parameter variance estimates. Plots for MSE, bias, and coverage are available in the appendix. Compared to the HM-RE model, the RE model demonstrated greater MSE and upward bias in the variance components, especially when $\mathrm{K}$ was small, regardless of the invariance condition. Although 95\% HDI posterior intervals are not designed to strictly provide $95 \%$ frequentist coverage, both models generally achieved $95 \%$ coverage.
Notable exceptions were latent score estimates (.906, .926 for RE and HM-RE, respectively), all parameters when full non-invariance is present (.85-.93, .907-.923, respectively), and random effects when invariance is present (coverage of 1). The RE model demonstrated decreased coverage on the group variance estimates, regardless of invariance condition (.912-.945). However, these differences were minor and both models performed similarly.

More importantly, the decision rates and accuracy were markedly different between the two approaches (See figure 2). While HM-RE and RE generally demonstrated high positive (variance present) and negative (variance absent) predictive value, the HM-RE model uniformly permitted more decisions while maintaining conditional accuracy. Therefore, the HM-RE model permitted uniformly more accurate decisions about invariance and non-invariance.

Constant non-invariance was the only invariance condition in which the HM-RE did not outperform RE in decision accuracy; in this particular scenario, there is no shared information across parameters or items for the inclusion model to utilize, and therefore the decision rates are equivalent between models. Outside of the constant condition, the difference in decision rates ranged from a mere $.04(n=50$, 5 items non-invariant, $K=3, K=5)$ to a surprising .90 ( $n=50$, full invariance, $\mathrm{K}=3, \mathrm{~K}=5$ ) in favor of the HM-RE model.

Unlike the RE model, the hierarchical inclusion model explicitly permits variance to be effectively removed from the model, which permits HM-RE to quickly accumulate posterior evidence in favor of invariance (See figure 3 for an example posterior). Confirming this, the models primarily differed in their capacity to accumulate evidence in favor of invariance. Notably, the true negative rate increased across sample size at a greater rate under HM-RE than RE on the same data, without sacrificing performance in true positive rates or estimation accuracy (See appendix).

\section{Example}

In order to illustrate the use of the HM-RE model and to compare its estimates to the RE model, we used an openly available data set. The example data was derived from the open psychometrics Big Five Inventory dataset (Open Source Psychometrics Project, 2014). Here, the goal was to determine whether the agreeableness scale is invariant across various racial categories.

From this dataset, the ten agreeableness scale items and race were extracted. Rows with missing data or race marked as "other" were removed. A random subsample of 1500 was drawn from the four largest race categories (European, Mixed, Indian, Southeast Asian), and included with all other observations from other race categories (Arctic, Middle Eastern, North African, Indigenous Australian, Native American, North East Asian, Pacific, West African). The final sample consisted of 3,163 observations from 12 different 

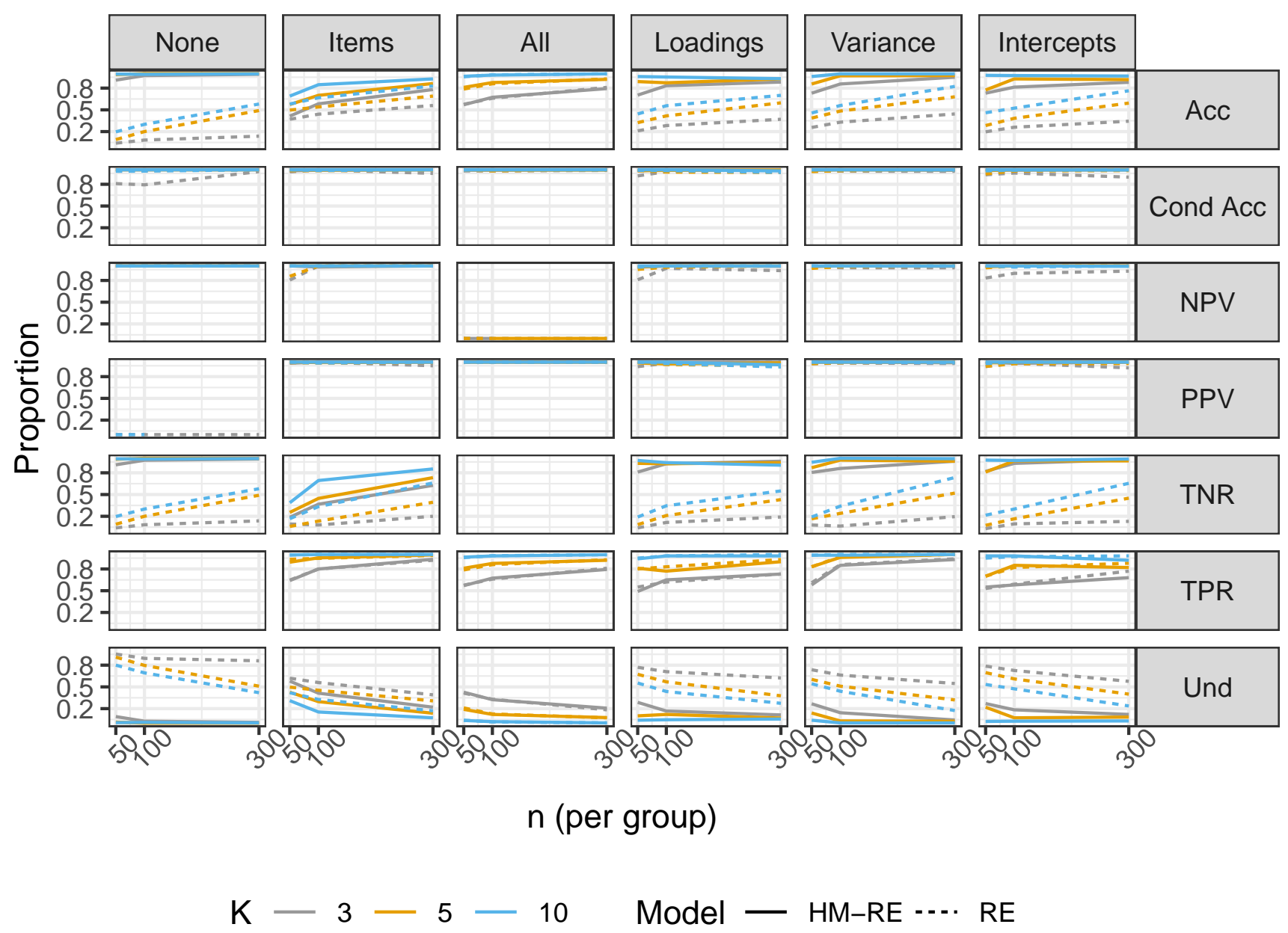

Figure 2. Decision accuracy rates across simulation conditions. "None": No variance; "Items": 5/10 items have variance in parameters; "All": All parameters vary; "Loadings": Lambda coefficients vary; "Variance": (Log) residual variances vary; "Intercepts": Intercepts vary. Acc(uracy) is the proportion of all possible decisions that were correct. Cond(itional) Accuracy is the proportion of decisions made that were correct. NPV/PPV: Of the invariance and variance decisions, the proportion that are correct. TNR/TPR: Of all the possible invariance and variance decisions, the proportion detected. Und(ecided): The proportion of possible decisions which did not reach the BF threshold of $\frac{1}{6}$ or 6 .

race categories. Reverse-scored items were placed on the forward scale, and the data were standardized across groups for easier prior elicitation.

The proposed model and a random effects model were both fit to the data. The estimates for the variance components (available in Table 1) demonstrates the similarity between the RE and HM-RE models. The largest difference between the two models was in the decision making power. The HM-RE model permitted six decisions, out of 10 , to be made about MI of the item loadings while the RE model only resulted in 3. At the same time, the HM-RE model resulted in only one fewer decision about item intercepts ( 4 vs. 5). The omnibus SD-BFs (with a threshold ratio of 6) suggest that neither scalar nor strict invariance is tenable. Of course, the magnitudes of the variances may be taken into account when deciding whether measurement invariance is practically acceptable.

Both models can examine every possible pairwise difference across groups' item parameters. The number of possible comparisons is $1.5 K(K-1) J=1980$. Although this number of comparisons would raise concerns of multiplicity and inflated false positive rates, the hierarchical structure attenuates the problem of multiple comparisons (Gelman et al., 2012). Additionally, the evidentiary threshold for the Bayes factor is set to 6 or $\frac{1}{6}$, which is a reasonably strict threshold. Of these 1980 comparisons, the HM-RE model suggested 949 comparisons to be sufficiently evidentiary, compared to the 777 comparisons suggested by the 


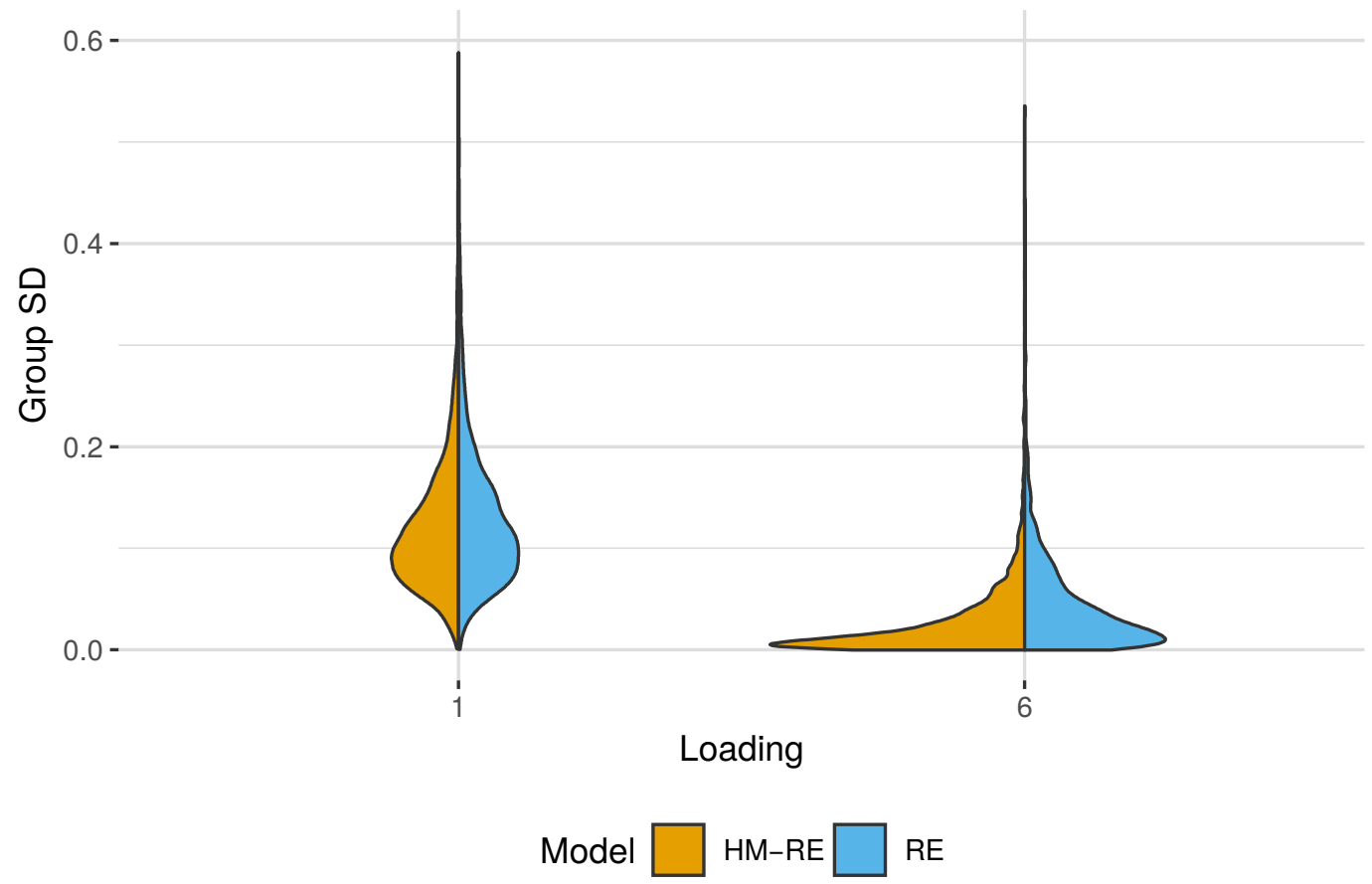

Figure 3. Example posterior distributions for one non-invariant (item 1) and one invariant (item 6) loading (K=5, n=100) under the two models. Note that the density at $\sigma_{\lambda, 6}=0$ is notably higher under the HM-RE model than under the RE model.

Table 1

Estimated Parameter Standard Deviations

\begin{tabular}{rllllll}
\hline \multicolumn{2}{c}{$\sigma_{\lambda}$} & \multicolumn{2}{c}{$\sigma_{\sigma}$} & \multicolumn{2}{c}{$\sigma_{v}$} \\
Item & HM-RE & RE & HM-RE & RE & HM-RE & RE \\
\hline 1 & $0.067(0.141)$ & $0.075(0.347)$ & $0.119(0.000)$ & $0.126(0.000)$ & $0.201(0.000)$ & $0.211(0.000)$ \\
2 & $0.028(7.128)$ & $0.039(3.398)$ & $0.053(0.031)$ & $0.058(0.015)$ & $0.106(0.004)$ & $0.114(0.000)$ \\
3 & $0.067(.874)$ & $0.084(0.409)$ & $0.052(0.630)$ & $0.059(0.380)$ & $0.075(1.838)$ & $0.085(1.787)$ \\
4 & $0.026(8.003)$ & $0.037(4.041)$ & $0.166(0.000)$ & $0.178(0.000)$ & $0.034(2.577)$ & $0.041(2.261)$ \\
5 & $0.019(10.584)$ & $0.027(6.626)$ & $0.110(0.001)$ & $0.123(0.001)$ & $0.046(4.730)$ & $0.046(1.273)$ \\
6 & $0.041(3.510)$ & $0.057(9.856)$ & $0.044(.840)$ & $0.052(1.135)$ & $0.061(.834)$ & $0.071(0.305)$ \\
7 & $0.055(1.856)$ & $0.069(0.612)$ & $0.095(0.000)$ & $0.105(0.001)$ & $0.132(0.002)$ & $0.137(0.001)$ \\
8 & $0.016(12.253)$ & $0.024(7.312)$ & $0.075(0.005)$ & $0.083(0.007)$ & $0.035(4.610)$ & $0.039(3.538)$ \\
9 & $0.034(7.043)$ & $0.051(1.576)$ & $0.034(2.432)$ & $0.044(2.336)$ & $0.067(0.148)$ & $0.078(0.050)$ \\
10 & $0.053(3.386)$ & $0.078(0.825)$ & $0.021(6.660)$ & $0.024(6.647)$ & $0.075(0.249)$ & $0.086(0.031)$ \\
\hline Decisions & 6 & 3 & 7 & 7 & 4 & 5 \\
MI & 5 & 3 & 1 & 6 & 0 & 0 \\
Non-MI & 1 & 0 & 6 & 6 & 4 & 5 \\
\hline
\end{tabular}

Note. Estimated standard deviations of each parameter for each item, for both the hierarchical inclusion random effects (HM-RE) model and the random effects (RE) model. Values in parentheses are approximate $\mathrm{BF} 01$ estimates in favor of measurement invariance. Decisions are based on whether $\mathrm{BF}_{01}>=6$ or $<=\frac{1}{6}$. 


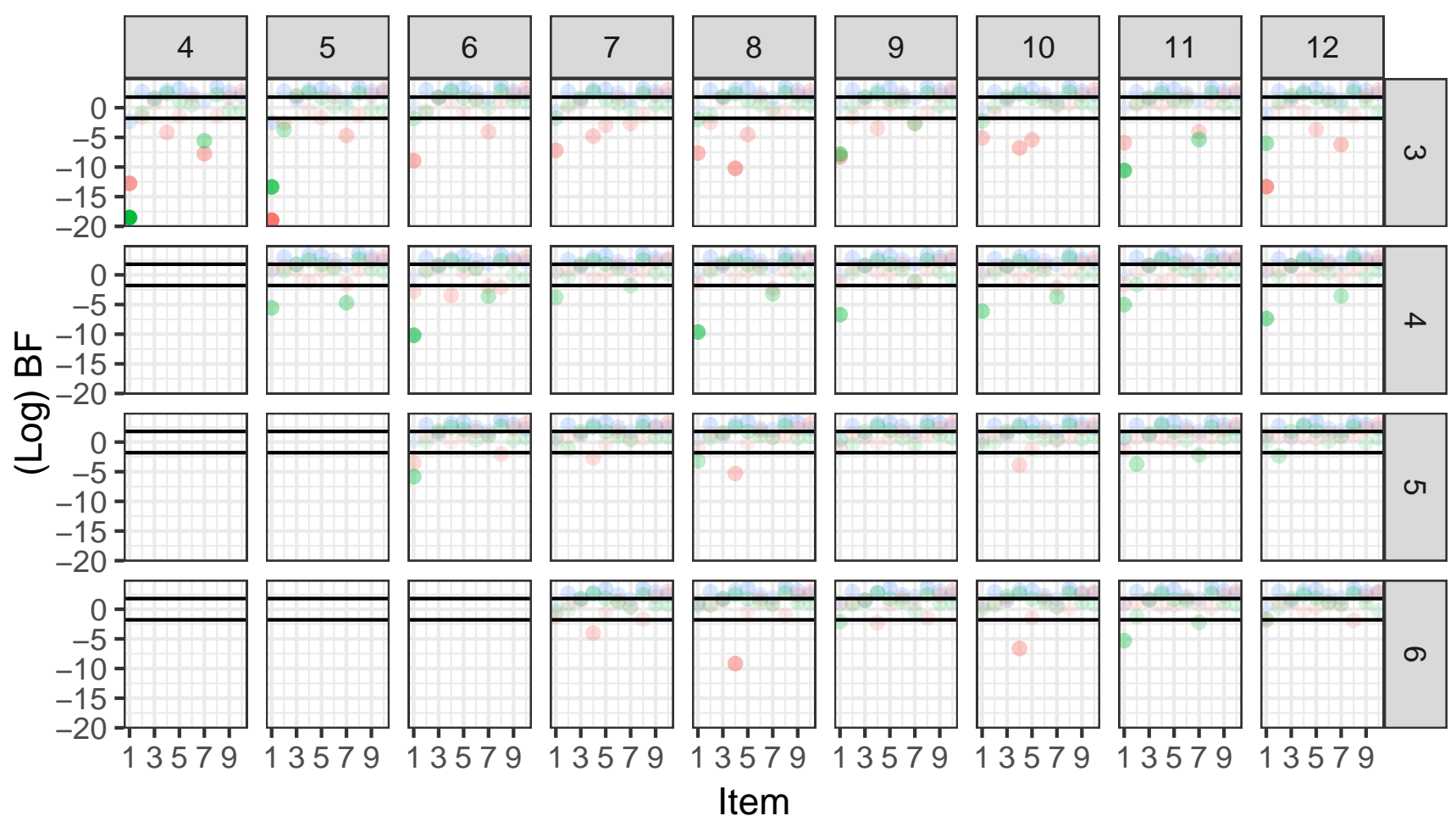

Parameter $\bullet \quad($ Log) SD $\bullet$ Intercept $\bullet$ Loading

Figure 4. Log BF01 values from a subset of all pairwise comparisons. The subset contains four groups with the greatest number of non-invariant parameters. Black lines correspond to $\log (6)$ and $\log (1 / 6)$. Low (negative) BF values suggest noninvariance. The groups are coded in the rows and columns as follows. 3: European; 4: Indian; 5: Middle Eastern; 6: North African; 7: Ind. Australian; 8: Native American; 9: NE Asian 10: Pacific; 11: SE Asian; 12: West African.

RE model. All BFs can be plotted for ease of interpretibility but given the larger number, we present a subset in Figure 4 as an example. The plot suggests a pattern of difference in residual variance between Caucasian Europeans and other ethnicities for several items. Likewise, a pattern of difference in intercepts for items 1 and 7 is observable between Indian respondents and other groups.

\section{Discussion}

Measurement invariance assessment is a critical step in psychological research. Without assessing or accounting for the presence of non-invariance, inferences about latent differences and relationships is difficult or impossible. One approach is to use a random effects model to account for variance in the measurement model. The random effects approach affords many advantages over the traditional approach, but can be improved. This paper evaluated an augmented random effects model called HM-RE. Specifically, the HM-RE model includes a hierarchical inclusion model on the random effect variance components. The HM-RE improves the RE model by modelling invariance, and by pooling invariance information across parameters with shared characteristics (e.g., parameters of the same item, parameters of the same type). By doing so, more decisions can be made about the presence of non-invariance and invariance, without sacrificing estimation accuracy. The improved efficiency is particularly useful in samples common to psychological research (e.g., few groups and small sample sizes).

The HM-RE can be used by applied researchers for a number of different purposes. Applied researchers who wish to assess which items are invariant may use the HM-RE as a relatively decisive tool for doing so. If the goal is to assess predictors of non-invariance, additional shared characteristics can be included in the inclusion model. The HM-RE can suggest which parameters are effectively invariant and can be fixed, if a simpler model is desired. Importantly, because the random effects approach does not require full nor partial invariance for meaningful group comparisons (de Jong et al., 2007), one may include the HM-RE measurement model in 
broader structural equation models.

The HM-RE as presented here only supports unidimensional models with no residual covariances, but it is not limited to this. As is possible with the RE, this can be extended to include multiple latent factors, and residual covariances through the phantom variable approach (Merkle \& Rosseel, 2018). Additionally, the presented model assumes a normal response likelihood, but the HM-RE can be used with IRT and other likelihoods. In IRT models, the hierarchical inclusion model would be imposed on the discrimination and threshold variances.

We only discussed a two level, random measurement model with no covariates. The HM-RE can accommodate multiple nested or crossed random factors and continuous group-level covariates (E.g., see A. J. Verhagen \& Fox, 2012); the inclusion model would then additionally predict the prior scales for the added variance terms and coefficients.

\section{References}

Barnard, J., McCulloch, R., \& Meng, X.-L. (2000). Modeling Covariance Matrices in Terms of Standard Deviations and Correlations, with Application to Shrinkage. Statistica Sinica, 10(4), 1281-1311.

Carpenter, B., Gelman, A., Hoffman, M. D., Lee, D., Goodrich, B., Betancourt, M., ... Riddell, A. (2017). Stan : A Probabilistic Programming Language. Journal of Statistical Software, 76(1). doi:10.18637/jss.v076.i01

Cheung, G. W. \& Rensvold, R. B. (1999). Testing Factorial Invariance across Groups: A Reconceptualization and Proposed New Method. Journal of Management, 25(1), 1-27. doi:10.1177/014920639902500101

Cheung, G. W. \& Rensvold, R. B. (2002). Evaluating Goodness-of-Fit Indexes for Testing Measurement Invariance. Structural Equation Modeling: A Multidisciplinary fournal, 9(2), 233-255. doi:10 . 1207 / S15328007SEM0902\{\_\}5

de Jong, M. G. \& Steenkamp, J.-B. E. M. (2010). Finite Mixture Multilevel Multidimensional Ordinal IRT Models for Large Scale Cross-Cultural Research. Psychometrika, 75(1), 3-32. doi:10.1007/s11336-009-9134-z

de Jong, M. G., Steenkamp, J.-B. E. M., \& Fox, J.-P. (2007). Relaxing Measurement Invariance in Cross-National Consumer Research Using a Hierarchical IRT Model. Journal of Consumer Research, 34(2), 260-278. doi:10. $1086 / 518532$

Deng, H. \& Wickham, H. (2011). Density estimation in R. Retrieved from http://www2.cs.uh.edu/ ceick/7362/T24.pdf

Dickey, J. M. \& Lientz, B. P. (1970). The Weighted Likelihood Ratio, Sharp Hypotheses about Chances, the Order of a Markov Chain. The Annals of Mathematical Statistics, 41(1), 214-226. doi:10.1214/aoms/1177697203
Gelman, A., Hill, J., \& Yajima, M. (2012). Why We (Usually) Don't Have to Worry About Multiple Comparisons. Fournal of Research on Educational Effectiveness. doi:10.1080/19345747.2011.618213

Gelman, A. \& Loken, E. (2013). The garden of forking paths: Why multiple comparisons can be a problem, even when there is no 'fishing expedition' or 'p-hacking'. Retrieved from http : / / www . stat . columbia . edu / $\sim$ gelman/research/unpublished/p_hacking.pdf

Hedeker, D., Mermelstein, R. J., \& Demirtas, H. (2008). An application of a mixed-effects location scale model for analysis of ecological momentary assessment (EMA) data. Biometrics, 64(2), 627-634. doi:10.1111/j.15410420.2007.00924.x

Hoffman, M. D. \& Gelman, A. (2011). The No-U-Turn Sampler: Adaptively Setting Path Lengths in Hamiltonian Monte Carlo. Retrieved from http://arxiv.org/abs / 1111.4246

Lewandowski, D., Kurowicka, D., \& Joe, H. (2009). Generating random correlation matrices based on vines and extended onion method. Fournal of Multivariate Analysis, 100(9), 1989-2001. doi:10.1016/j.jmva.2009.04.008

Meade, A. W. \& Wright, N. A. (2012). Solving the measurement invariance anchor item problem in item response theory. Journal of Applied Psychology, 97(5), 1016-1031. doi:10.1037/a0027934

Meredith, W. (1993). Measurement invariance, factor analysis and factorial invariance. Psychometrika, 58(4), 525543. doi:10.1007/BF02294825

Meredith, W. \& Teresi, J. A. (2006). An essay on measurement and factorial invariance. Medical Care, 44(11), S69-S77. doi:10.1097/01.mlr.0000245438.73837.89

Merkle, E. C. \& Rosseel, Y. (2018). blavaan : Bayesian Structural Equation Models via Parameter Expansion. Fournal of Statistical Software, 85(4). doi:10.18637/jss.v085. i04

Open Source Psychometrics Project. (2014). Open psychology data: Raw data from online personality tests. Retrieved from https : / / openpsychometrics . org / _rawdata/

Rast, P. \& Ferrer, E. (2018). A Mixed-Effects Location Scale Model for Dyadic Interactions. Multivariate Behavioral Research, 1-20. doi:10 . 1080 / 00273171 . 2018 . 1477577

Rast, P., Hofer, S. M., \& Sparks, C. (2012). Modeling Individual Differences in Within-Person Variation of Negative and Positive Affect in a Mixed Effects Location Scale Model Using BUGS/JAGS. Multivariate Behavioral Research, 47(2), 177-200. doi:10.1080/00273171. 2012.658328

Steenkamp, J.-B. E. M. \& Baumgartner, H. (1998). Assessing Measurement Invariance in Cross-National Con- 
sumer Research. Journal of Consumer Research, 25(1), 78-107. doi:10.1086/209528

Verhagen, A. J. \& Fox, J.-P. (2012). Bayesian tests of measurement invariance. British fournal of Mathematical and Statistical Psychology, 66(3), 383-401. doi:10.1111/ j.2044-8317.2012.02059.x

Verhagen, J., Levy, R., Millsap, R. E., \& Fox, J.-P. (2016). Evaluating evidence for invariant items: A Bayes factor applied to testing measurement invariance in IRT models. fournal of Mathematical Psychology, 72, 171182. doi:10.1016/j.jmp.2015.06.005

Wagenmakers, E.-J., Lodewyckx, T., Kuriyal, H., \& Grasman, R. (2010). Bayesian hypothesis testing for psychologists: A tutorial on the Savage-Dickey method. Cognitive Psychology, 60(3), 158-189. doi:10.1016/j. cogpsych.2009.12.001

Williams, D. R., Liu, S., Martin, S. R., \& Rast, P. (2019). Bayesian Multivariate Mixed-Effects Location Scale Modeling of Longitudinal Relations among Affective Traits, States, and Physical Activity. PsyArXiv. doi:10. 31234/osf.io/4kfjp 
Appendix

Additional Figures

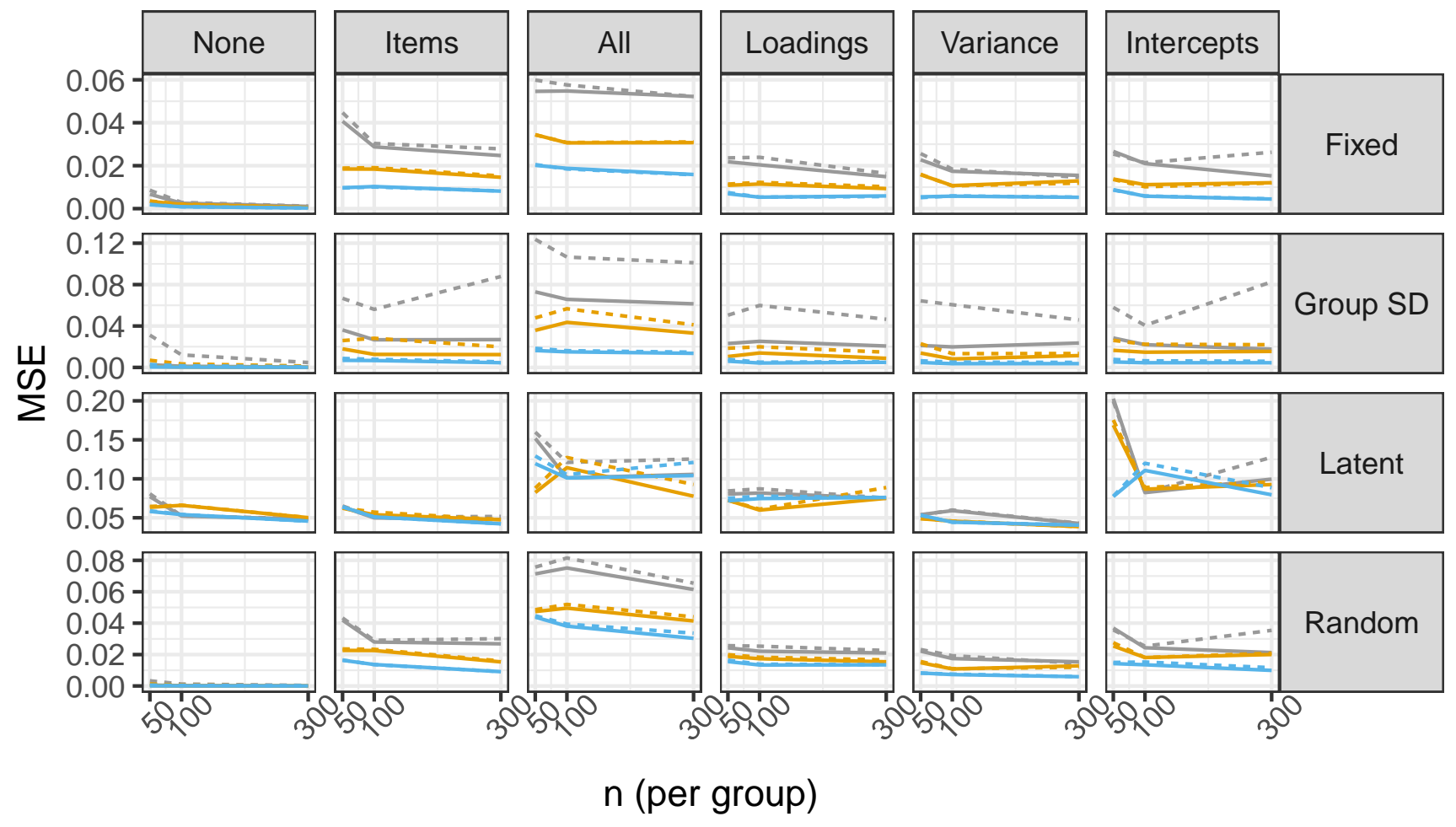

$$
\mathrm{K}-3-5-10 \quad \text { Model }-\mathrm{HM}-\mathrm{RE} \cdots \mathrm{RE}
$$

Figure A1. Mean squared errors per simulation condition, for each type of parameter. "None": No variance; "Items": 5/10 items have variance in parameters; "All": All parameters vary; "Loadings": Lambda coefficients vary; "Variance": (Log) residual variances vary; "Intercepts": Nu parameters vary 


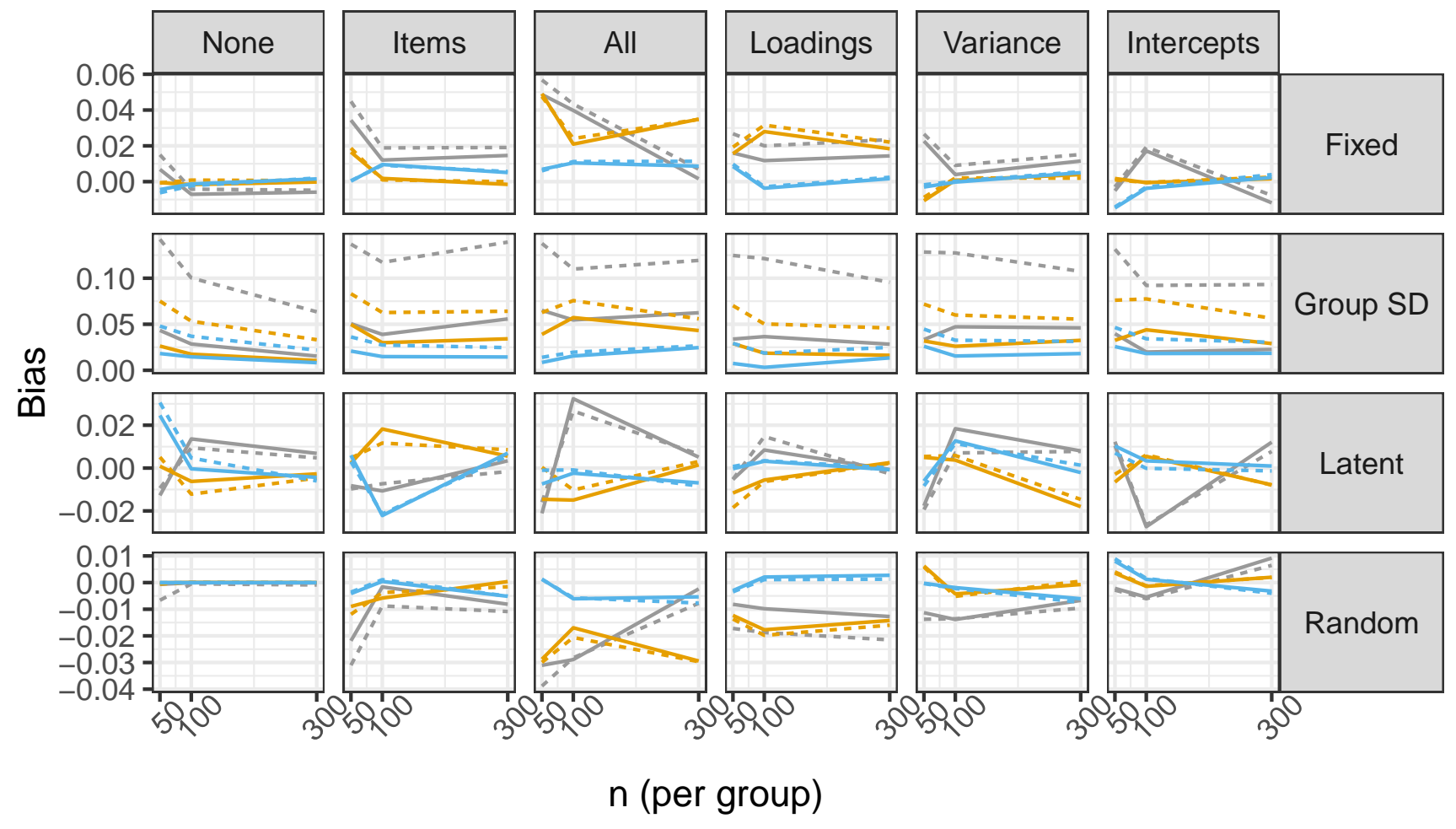

$\mathrm{K}-3-5-10 \quad$ Model $-\mathrm{HM}-\mathrm{RE} \cdots \mathrm{RE}$

Figure A2. Raw bias estimates per simulation condition, for each type of parameter. No scaling was performed, and these estimates are intended for directional bias assessment only. 


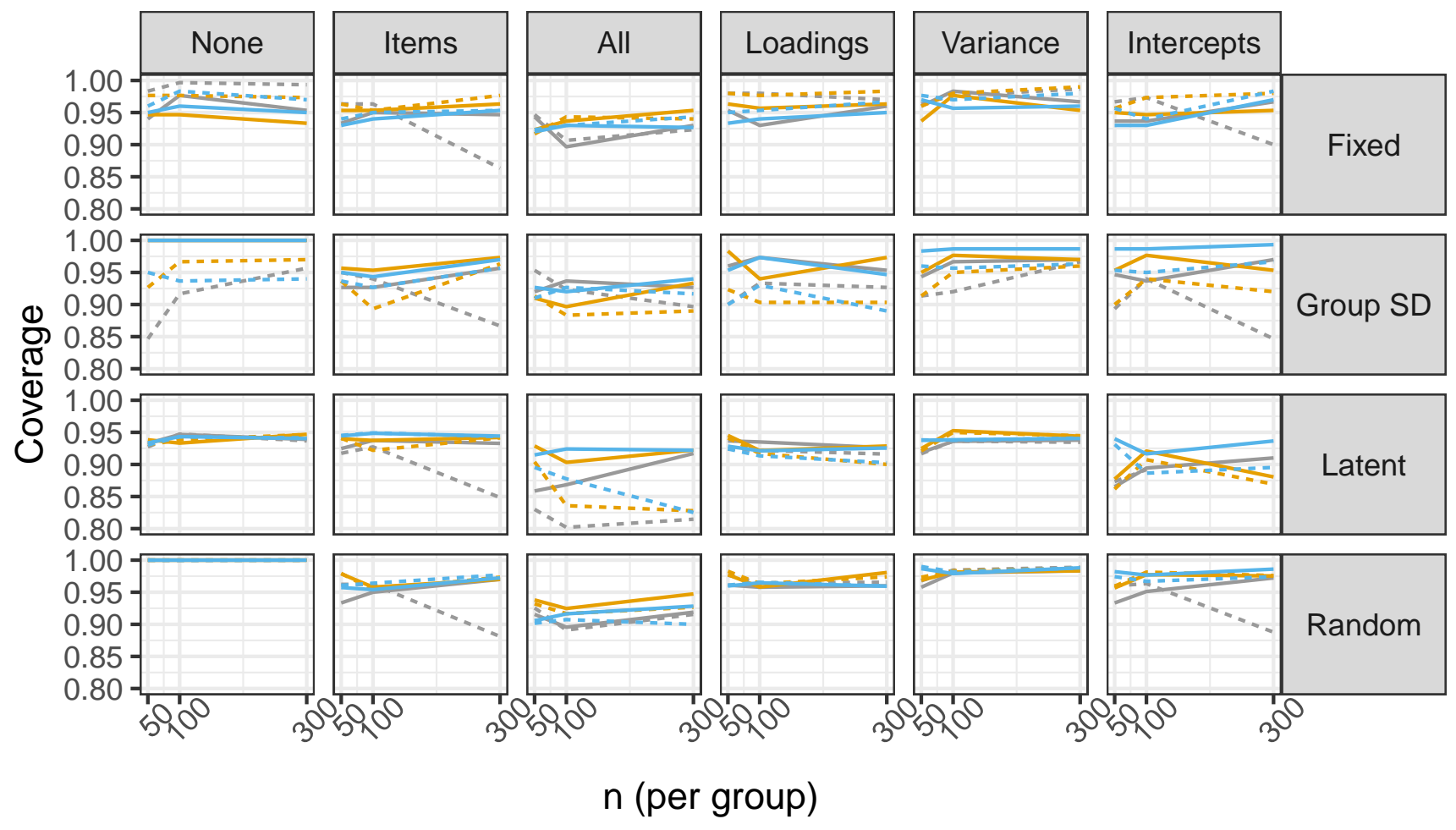

$$
\mathrm{K}-3-5-10 \quad \text { Model }-\mathrm{HM}-\mathrm{RE} \cdots \mathrm{RE}
$$

Figure A3. Coverage estimates per simulation condition, for each type of parameter. 95\% continuous highest density intervals were computed per parameter. For each iteration, the proportion of true values within the HDI was computed. The means of those proportions are plotted here. 


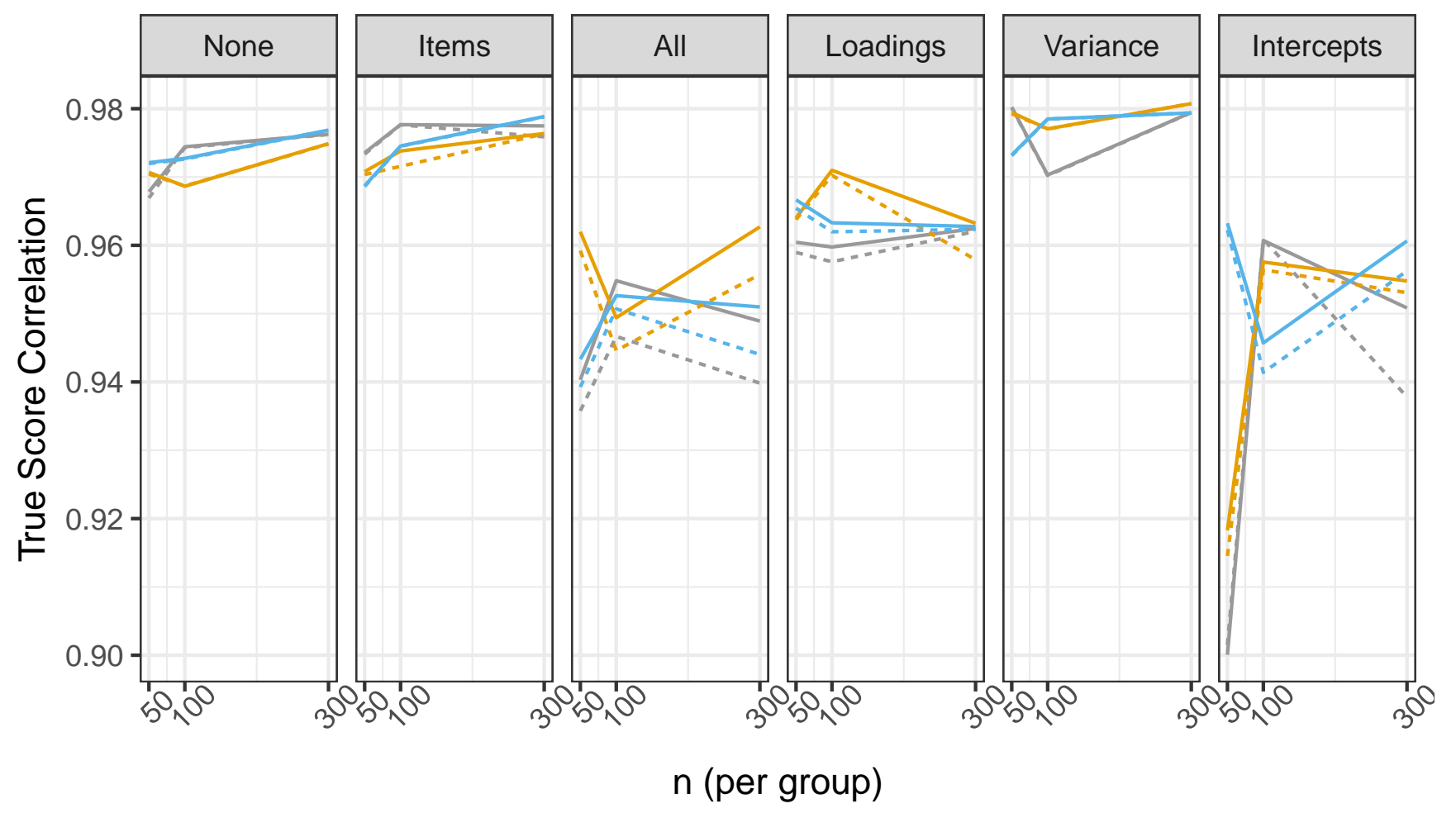

$\mathrm{K}-3-5-10 \quad$ Model $-\mathrm{HM}-\mathrm{RE} \cdots \mathrm{RE}$

Figure A4. Correlation between true and estimated factor scores per simulation condition. 